\title{
LA INTEGRACIÓN LATINOAMERICANA COMO VOCACIÓN. EL CASO DE LEONARDO JEFFS CASTRO (†)
}

\author{
LATIN AMERICAN INTEGRATION AS A VOCATION. THE CASE \\ OF LEONARDO JEFFS CASTRO (†)
}

\author{
1938-2015 \\ In memoriam
}

Martín Lara*

El presente estudio busca rescatar el aporte a la integración latinoamericana que se ha gestado desde el mundo de la academia durante la segunda mitad del siglo XX. En particular, se trabajará dicha materia sobre los aportes desarrollados por el profesor Leonardo Jeffs Castro $(\dagger)$. Considerando ciertas variables, se realizará un breve repaso a su vida profesional, enfocándonos en un análisis de sus primeros trabajos en la materia, la evolución conceptual de sus investigaciones, hasta llegar a su aproximación teórica y orientaciones temáticas de sus ponencias en congresos acerca de la materia. Finalmente, se sostendrá mediante varios argumentos que la labor del profesor fomentó el diálogo de disciplinas entre naciones y coadyuvó en el proceso de integración de países hermanos.

Palabras claves: Leonardo Jeffs, integración latinoamericana, relaciones internacionales, Bolivia.

This study seeks to rescue the contribution to Latin-American integration, originated from the academic circles during the second half of the XXth century. Said subject will be analysed according to the contributions of professor Leonardo Jeffs Castro ( $\dagger$ ). Considering a series of variables, a brief summary of his professional life will take place, focusing on the analysis of his first works on the subject, the conceptual evolution of his research, arriving to his theoretical approximation and thematic orientations of his presentations in conferences on the matter. Finally, through a series of arguments it will be stated that the works of professor Jeffs encouraged the dialogue between disciplines among nations and supported the process of integration among fellow nations.

Key words: Leonardo Jeffs, Latin American integration, international affairs, Bolivia.

\section{Palabras iniciales}

Resulta complejo escribir acerca de personas conocidas y cercanas, más todavía cuando hemos sido testigos de su partida. Pero esta dificultad se transforma en desafío y en una oportunidad, en la medida que el recuerdo fragmentario se sistematiza, permitiendo hilvanar destellos del pasado que en la forma de conversaciones, anécdotas y frases -por muy sutiles o intrascendentes en su momento-que con el paso del tiempo se pueden comprender y valorar por su importancia y significado. Dentro de este marco, me esforcé por situar dichos recuerdos en un cuadro mayor que permitieran analizar la trayectoria profesional de quien ya partió y su aporte al estudio de la integración latinoamericana.

Entender la obra del profesor Leonardo Jeffs Castro sirve para conceptualizar el esfuerzo de una generación de académicos por insertar un debate de ideas e ideales en el plano de la academia que, a su vez, apunta a largas lecturas que tienen sus antecedentes en las profundidades de la historia republicana de los países americanos e incluso más atrás. Esto lo decimos a propósito que la génesis de la integración latinoamericana tiene sus primeros antecedentes en las postrimerías del siglo XVIII, al alero de la Ilustración, y que en sujetos como Francisco de Miranda, Simón Rodríguez y Carlos Montúfar, dio el impulso para que se fuera consolidando a lo largo del siglo XIX con la gestación de las independencias en un primer momento y el surgimiento del Estado-Nación en una segunda parte. Ambas etapas se enmarcaron en nociones genéricas, que buscaban en la afinidad cultural y similitud política de los Estados, espacios de diálogo y proyecto de un futuro compartido, cuya síntesis conceptual la dio José Artigas con su preclara concepción de Patria grande (Bolívar y Cuéllar 2007).

\footnotetext{
* Universidad Católica Silva Henríquez, Santiago, Chile. Correo electrónico: martinlara@uc.cl
} 
Sin embargo, es en el siglo XX cuando esta idea de integración latinoamericana da paso a un concepto denso que desplaza la noción política y de unidad territorial a una de tipo social, económica y neocultural, cuya senda se va complicando a medida que disciplinas como la filosofía, sociología, ciencia política e historia adquieren un sentido explicativo de la realidad. Así, discursos propios que prefigurarán en autores como José Carlos Mariátegui, Frantz Fanon, Leopoldo Zea, Arturo Andrés Roig, Walter Mignolo, Enrique Dussel, y en nuestro caso bajo las circunstancias de vida y singular alcance profesional de Leonardo Jeffs, darán una concepción autónoma, diversa y auténtica. Con ello, entendemos que aproximadamente en un arco de tiempo de setenta años, la concepción de integración latinoamericana fue madurando y consolidándose, teniendo un cariz propio y fundamentalmente académico.

\section{La vocación en forma: Historia de las relaciones internacionales}

Con todo, no podemos referirnos a la idea de integración sin previamente comprender que esta tiene su antesala en la labor del profesor Jeffs en sus estudios de la historia de las relaciones internacionales, que la entendía como "el quehacer de los diversos actores que intervienen en las relaciones internacionales a través del tiempo y como la disciplina que los estudia" (Jeffs 2014). La vinculación de oficio con la historia de las relaciones internacionales se manifestó en sus propias palabras desde tres dimensiones: sus intereses personales gestados en el Instituto Pedagógico de la Universidad de Chile a fines de la década del 50; como actor de las relaciones internacionales en la década del 70; y finalmente como estudioso de la disciplina en la década del 90 (Jeffs 2014:1). El interés de esta área del conocimiento pensamos que surge en su persona por algunas variables que son concomitantes con su generación. Una de ellas, es el notorio cuestionamiento en el mundo académico de la desigual relación diplomática que se daba entre los países de América Latina y Estados Unidos, con total nitidez tras la revolución cubana. En ese aspecto, sin desconocer la naturaleza ideológica en la figura del profesor, quien fue un conocido simpatizante con el pensamiento socialcristiano (Centro de Estudios Chilenos), la idea de imperialismo se presentaba no solo como una exageración de paternalismo político, sino también como un obstáculo de diálogo directo entre las cancillerías al sur del río Grande, debido a que en varias ocasiones Washington jugó como intermediario o garante en las diferencias entre países. Esto último se manifestó para que en 1971 cofundara el Instituto Chileno-Boliviano de Antofagasta (Solíz Rada 2013) y once años más tarde, bajo otras circunstancias, el CEDECH (Centro de Estudios Chilenos), cuyo nacimiento obedece a desestimar el debate de binomios izquierdas/derechas y democracia/ dictadura, optando por un "bloque patriótico", cuyo complejo planteamiento contiene ideas de origen nacionalista, marxista y socialcristiana (Centro de Estudios Chilenos 2016).

Un segundo punto que activó su vuelco hacia este tema fue su temprano interés por la historia económica ${ }^{1}$ que derivó hacia una mirada transnacional de los problemas económicos, de la que nacerá una premisa que alimentará su posterior pensamiento: que bajo profundas desigualdades económicas se genera no solo pobreza y tensión social entre la población de un país, sino también que promueve choque de intereses que exceden los límites nacionales, imposibilitando un diálogo fluido entre países de una misma región. Esta idea permite entender que su tesis de maestría presentada en el Instituto de Estudios Internacionales de la Universidad de Chile en 1982 se titulara Teoría y Práctica del aprismo sobre el imperialismo y la unidad de América Latina, y que sus trabajos posteriores acerca de la guerra del Chaco y la participación de chilenos en dicho conflicto, que se consolidó con su tesis doctoral titulada Chile y la Guerra del Chaco $(2011)^{2}$, giraran permanentemente en dicha línea de investigación.

Relacionado con lo anterior, y como tercer punto, están sus trabajos concernientes a las relaciones políticas y diplomáticas entre Chile y Bolivia, tema poco trabajado en nuestro país hasta ese momento ${ }^{3}$ y que lo convirtió en un experto en la materia, generando sólidas investigaciones entre las que se cuentan Aquiles Vergara Vicuña. Perfil biográfico de un hombre íntegro (1995), Encuentros y desencuentros: Chile y Bolivia 1928-1935 (2005) y Encuentros con Bolivia (2012). Justamente es Bolivia: su realidad, historia y su desvinculación con Chile, que lo llevarían a su difusión y tarea más preciada, la integración latinoamericana.

\section{Integración latinoamericana. Consolidación de una vocación}

Por su perfil profesional, la academia se convirtió en la "trinchera de ideas", en palabras de 
José Martí (Martí 1975: 15-23), y los seminarios, como su tan querido chileno-argentino ${ }^{4}$, en su punta de lanza. Claro está que siempre, desde todos los espacios posibles, pujó con discreción y respeto por lo que consideraba válido para el futuro de los países. Decimos "discreción" y "respeto", en tanto quienes lo conocimos somos testigos que el profesor Jeffs jamás hizo proselitismo político y menos intentó influenciar ideológicamente a los estudiantes; en aquel sentido, siempre se destacó por ser un profesional íntegro, con alto sentido del deber y un caballero en el plano personal. Para él, considerablemente influenciado por la Teoría de la Dependencia, el futuro de los países dependía de reconocer los "aspectos sociales del desarrollo económico" (Morales 2012: 237) que se sostenía en una democracia directa, con una participación ciudadana en los grandes debates, y un desarrollo económico que beneficiara a toda la población. En lo fundamental, la eliminación de la pobreza y la consolidación de una justicia social (Fernández 1996: 46) se convertirían en la base para un diálogo entre los países de la región.

Para quienes lo conocieron en el plano profesional, creo conveniente mencionar que la concepción de integración latinoamericana para Leonardo Jeffs, tiene una raíz mucho más profunda y no vinculada ciento por ciento con la academia, pues en su juvenil mirada cristiana y católica en la década de los 50 y 60 es donde, a nuestro juicio, se puede encontrar la génesis de su pensamiento latinoamericanista. Líder del movimiento Iglesia Joven, en conjunto con laicos y religiosos lucharon a fines de los sesenta por una Iglesia cercana a las personas y como reza en los principios de la organización, pujando por "la liberación del hombre, que es la condición primordial para construir el Reino de Dios" (Lara, 2015), planteando que el Santo Evangelio debía materializarse con una práctica real y sin aspavientos anquilosadas en las formas y lujos. Con solo dar un ejemplo de aquello, podemos mencionar lo que denunciaba dicha agrupación respecto de la complacencia de la Iglesia frente a:

La violencia provocada por los ricos y poderosos; La explotación del hombre por el sistema inicuo del lucro; El imperialismo internacional del dinero; El engaño de una falsa democracia manejada por unos pocos; La sumisión de las conciencias a través de los monopolios, propietarios de los medios de información; La segregación racial, cultural y económica; La instrumentalización de la educación en favor de las clases dirigentes y; La división del pueblo para consagrar su dominación (Echeverría 1993: 150 y ss).

Nos podemos dar cuenta que estas afirmaciones eran concordantes e influenciadas por varias ideas que orbitaban en la mente de los jóvenes de aquella época, entre las que se cuentan las propugnadas por el Concilio Vaticano II (1962-1965), la II Conferencia Episcopal Latinoamericana (Medellín, 1968), la Teología de la liberación y claros guiños con los planteamientos teóricos poscoloniales. En síntesis, como el mismo profesor diría, sus actos y pensamientos se dieron bajo el "signo de los tiempos" que le tocó vivir, pues "se trataba de un período marcado por la influencia de la Revolución Cubana, la Alianza para el Progreso, el surgimiento de gobiernos militares y la invasión norteamericana a Santo Domingo" (Jeffs 2012: 2), de esta manera se entiende que, en propias palabras de Jeffs, el Movimiento "creyó en el diálogo cristiano-marxista a través de la praxis" (Jeffs 2012: 7).

El viaje, como acto iniciático, fue preponderante en la germinación de su pensamiento latinoamericano. Gracias a una beca del gobierno de Estados Unidos financiada por la Universidad de Texas, en conjunto con otros seis líderes universitarios de la entonces Facultad de Humanidades y Educación de la Universidad de Chile, pudo conocer varias ciudades norteamericanas y también algunos países como México y Puerto Rico, siendo en estos últimos donde se dio cuenta de las notables similitudes culturales, pero sobre todo de las carencias materiales y espirituales. Más tarde, en 1969, y bajo otras circunstancias, hizo su primer viaje a Bolivia en el que rememora:

Fue para mí como si hubiera estado ciego y viera el mundo, fue sorprendente; fue como un impacto en mi ser, gente joven de ese país conocerla tan esperanzada, comprometida por un país mejor, con un espíritu tan abierto hacia todos incluso nosotros los chilenos. De allí empecé a interesarme por Bolivia (Rojas 2015).

Desde ahí en adelante, la historia es más o menos conocida, diciendo en sus propias palabras que 
a partir de todas estas experiencias, me he sentido un actor de las relaciones internacionales, que actuando conjuntamente con otros, hemos realizado una labor en pro de la integración latinoamericana (Jeffs, 2014: 6).

La lucha por la integración de América Latina la focalizó en acercar las relaciones entre Chile y sus países limítrofes. Por ejemplo, para él la exacerbación de la nación y el nacionalismo, en momentos en que los límites se están diluyendo a causa de los efectos de la globalización, obstruye directamente las posibilidades de la integración. Así, en una entrevista sostuvo que

\section{en Chile, Perú y Bolivia no deberían} celebrarse los aniversarios de las confrontaciones fratricidas, (pues) no ayudan a restañar las heridas del pasado y encarar mejor los acercamientos del presente (Solís Rada 2013).

En particular, mejorar y consolidar las relaciones con Bolivia fue a lo que dedicó mayor tiempo de su trabajo, reconociendo que el tema marítimo era el principal nudo. Férreo defensor de una salida soberana al Pacífico para Bolivia, en sus últimos años de vida generó con fuerza un discurso proclive a ello. Identificaba que el problema no solo dependía de acuerdos políticos entre los gobiernos, sino que un factor no menor era también la opinión pública y reconocimiento íntegro del problema por parte de la sociedad en general y, en este caso, la academia se convertía en un soporte fundamental e insoslayable. En la ciudad de La Plata en el 2012 planteaba:

También cabe un rol a las personas y a la sociedad civil. De una manera especial a las Universidades, las Iglesias, las organizaciones de los trabajadores, empresarios y estudiantes (...) Hay que conocer la historia de las relaciones, con sus luces y sombras, tal como lo han hecho los diplomáticos y escritores Walter Montenegro de Bolivia y el chileno Oscar Pinochet de la Barra, y, luego divulgarla (...) Por último tenemos la obligación de conocernos, porque así superaremos los prejuicios que existen entre nosotros, que no ayudan en nada a la solución del diferendo marítimo (Jeffs

2012: 12-13).

Cual círculo virtuoso, el proceso de integración se manifestaría en la medida que nos reconociéramos en el otro. Más de una vez afirmó que en Bolivia no solo estaba un pueblo hermano de Chile y de los demás países de América Latina, sino también un pueblo que en su población recuerda a nuestros ancestros. La posibilidad de viajar para compartir y dialogar en todas las instancias que se presentaran era algo que recomendaba, pues no solo era un ejercicio de crecimiento personal, sino también de mirarnos en el espejo buscando "provocar que se conozca al otro, los viajes son fundamentales. Para hablar de temas de integración hay que hablar con los vecinos donde hay problemas" (Parra 2002-2003: 227). Desde este punto recién se permitiría que la "reivindicación marítima de Bolivia esté más cercana (Soliz Rada 2013), proyectando y potenciando "una condición para mejorar las relaciones entre nuestros gobiernos y nuestros pueblos, desarrollando no solo la capacidad crítica, sino también debemos desarrollar la capacidad autocrítica" (Jeffs 2014:1) que diera pie para relaciones internacionales estables y con proyección, sustentadas en la confianza recíproca y la amistad sincera de su clase política y todos los conciudadanos.

Conocedor del oficio y práctica diplomática para desviar o dilatar temas y las reticencias de la alta política de los países en cuestión para tomar el toro por las astas; para él, la universidad como espacio de libertad, cuestionamiento y búsqueda de la verdad se convertía en un punto de fuga para la discusión. En una visita a Bélgica el 2006, decía al respecto:

Lo interesante de todo esto es la oportunidad de haberse reunido personas vinculadas al campo de las ciencias sociales, tanto de Bolivia, Chile y Perú, y haber llegado a una propuesta de consenso sobre un tema muy complejo y donde hay intereses cruzados (El Mostrador 2006).

Ocho años más tarde, siguiendo la misma línea, en Buenos Aires planteaba que

desde el mundo académico creemos que tenemos la obligación, el derecho a expresarnos porque no hay suficientes oportunidades 
dentro del país para plantearlo (...) Puede que no sea mayoritaria nuestra opinión, pero es una opinión que debe ser considerada (Radio Cooperativa 2015).

Esta idea, que superficialmente puede ser llamativa, refleja una profundidad sustantiva de su pensamiento, pues pocos meses antes de fallecer, en un homenaje que se le rindió en las VI Jornadas de Historia de las Relaciones Internacionales celebradas en la Universidad Técnica Federico Santa María, volvió sobre el tema diciendo

El grueso de la gente estima que las relaciones internacionales son de exclusividad de los gobiernos. Yo estimo que sí; un actuar importante de ellas es el estatal, pero hay otros actores, los de la sociedad civil, y en ese sentido, las universidades y nosotros los académicos también tenemos nuestra voz y tenemos que expresarla, (...) a favor tenemos haber estudiado estos temas, entonces podemos aportar para que una política exterior sea más conveniente para el país (Jeffs 2015).

Leonardo Jeffs, como hemos podido comprobar, consideraba a la integración latinoamericana como un tema mayor, que no solo respondía a cuestiones de índole político, social y económico, sino per se histórico. Más aún, me atrevería a decir que tenía plena conciencia que su labor tenía vocación histórica, no solo como testigo, sino también como protagonista, enlazando su propio esfuerzo con las raíces de nuestro pasado. Con su salud deteriorada, si bien no pudo asistir como uno de los firmantes del Acta de Buenos Aires, participó en el diálogo previo que se generó en dicho círculo, adscribiendo plenamente a uno de los postulados de dicho documento, al afirmar que

Finalmente, dado que la aspiración a la unidad americana es uno de los legados más valiosos de la Independencia, una agenda de integración trinacional, que incluya el reencuentro de Bolivia con el mar, es una de las tareas urgentes (Acta de Buenos Aires 2015).

Como una manera de dar sentido a sus ideales, fundó en el 2005 el Centro de Estudios
Latinoamericanos al alero de la Facultad de Humanidades de la Universidad de Valparaíso, que tuvo por objetivo transformarse en un espacio de diálogo y reflexión del pensamiento latinoamericanista. Más aún, para dar coherencia y difusión al quehacer académico de dicho centro, funda Estudios Latinoamericanos (Espinoza 2005: 85), revista que en sus primeros números cuenta con interesantes trabajos de connotados y nóveles investigadores. Esto último fue otra muestra de su generosidad para con los que recién comenzaban. Sin embargo, las dificultades presupuestarias por parte de la universidad, exceso de trabajo administrativo y de docencia del profesor Jeffs -muy común entre los profesores de las universidades de esta parte del mundo-, sumado a la falta de trabajo colaborativo por parte de los integrantes de la unidad académica más cercana a dicho centro, hizo que la revista que nació llena de ímpetu, al poco andar dejara de tener el ritmo de edición deseado.

\section{Palabras finales}

El estudio de la historia de las relaciones internacionales como la idea de integración de los países americanos se desarrollaron de forma paralela y entrelazada, muchas veces sosteniéndose mutuamente; vinculadas de manera orgánica en su pensamiento que proyectó de manera nítida y elocuente en los textos publicados y de manera locuaz en los seminarios y congresos en distintas partes de la región. Aquí las distinguimos solo como un ejercicio, como una forma de diferenciar conceptos de naturaleza distinta, entendiendo a la primera como una disciplina y a la segunda como una doctrina.

Podemos afirmar que la idea de integración latinoamericana en la figura de Leonardo Jeffs si bien tiene un perfil decididamente académico, sus antecedentes provienen de su experiencia vital y las circunstancias históricas que le tocó vivir. El decidido y comprometido catolicismo personal que lo acompañó toda su vida, desde los años de escolar, sin duda es un aspecto ineludible en la formación de su concepto respecto de la integración. Acerca de este punto, recordemos que el Concilio Vaticano II marcó un cambio de época para los cristianos observantes de todo el mundo, impulsando en pleno contexto de Guerra Fría la idea del multilateralismo y legitimidad de las organizaciones internacionales, a tal punto que dichas instituciones fueron 
consideradas beneméritas del género humano (Allard 2015: 23). Claramente, un joven católico y líder de un movimiento laico como Iglesia Joven, en dicho Concilio veía una verdadera luz de esperanza hacia un mundo de mayor justicia y paz social.

Otro aspecto que genera su cercanía e impulso en la integración, lo dan las vivencias profesionales como clases, lecturas y viajes que pudo desarrollar por más de cincuenta años, generando una profundidad reflexiva que permitió macerar ideas y planteamientos, muchas veces no compartidos por el establishment de sus colegas, pues iban generalmente a contramano de ideas formativas imperantes en Chile como el patriotismo y excepcionalidad histórica por un lado, y el nacionalismo y emotivismo boliviano, por el otro (González y Ovando 2016: 58).

En esas oportunidades de distensión, que solo tienden a darse con relativa facilidad en los congresos fuera del país, como anécdota me comentó que era sobrino nieto de Leopoldo Zea, a quien de visita brevemente en su casa de la Ciudad de México, por agendas cruzadas, no tuvo la oportunidad de conocer, pero que sin duda pudo leer gracias a sus innumerables textos que lo hicieron participar de la gran corriente latinoamericanista que en México y Argentina se desarrollaba en la década de 1940 y 1950 (Saladino \& Santana 2003:277). ¿Habrá influido dicho vínculo familiar con su futura vocación? ¿Qué factores influyeron para pasar de un catolicismo conservador de sus primeros años a uno liberal e integracionista? ¿Interpretó el acuerdo de Charaña como una posibilidad real de integración? ¿Su concepción de la historia de las relaciones internacionales e integración latinoamericana obstaculizó de algún modo el desarrollo de su carrera profesional en los primeros años? ¿Cuántas vocaciones por los estudios latinoamericanos generó a lo largo de su pesado peregrinaje laboral por distintas universidades? ¿A cuánto tiempo estamos que se logré finalmente su anhelo más deseado?

Hombre de servicio público tuvo en la educación su principal medio de sustento. Escuelas de alta vulnerabilidad social, colegios privados, institutos profesionales y universidades de Santiago, Valparaíso y distintas regiones del país, fueron testigos de un hombre que se caracterizó por su bondad, generosidad y ausencia de rencores
(Boggia y Bravo 2016). También fueron espacios donde demostró ser, apropiándonos de palabras de su amigo Pedro Godoy, un "chileno excepcional y un iberoamericanista sin recovecos" (Godoy 2015), reconociéndose por ser comprometido con sus ideas y respetuoso de la diversidad de opciones, credos y orientaciones. Para el profesor, la educación era un medio no solo para alcanzar la dignidad material y espiritual que toda persona debiera poseer, sino el primer escalafón para la integración social, nacional y regional. No es casual entonces, que muchos exalumnos que pasaron por sus aulas lo recordemos como un profesor cálido, afable, respetuoso, cercano, siempre dispuesto a escuchar y, cuando la ocasión lo ameritaba, a dar un consejo atinado. Así, para él, la integración partía desde el más pequeño espacio, la sala de clases.

No puedo dejar de terminar estas palabras, apropiándome de un sentido homenaje del profesor Paz Rada de la Universidad Mayor de San Andrés, quien al recordar al Maestro y Amigo escribió:

"Leonardo acompañaba con su comprensión, palabras y amistad, las lágrimas que un boliviano derramaba al ver el inmenso mar desde los cerros de Valparaíso, encontraba, con paciencia y calma, las palabras pausadas, comprometidas y precisas, para describir y analizar los problemas y los procesos de nuestra historia común; su compromiso social y educativo tenía sus orígenes en la Doctrina Social de la Iglesia y en la militancia con los pobres y marginados de nuestras tierras" (Paz Rada, 2015).

\section{Agradecimientos}

Algunas ideas de este documento fueron adelantadas y comentadas en el XIII Seminario Argentino Chileno y VI Seminario Cono Sur de Ciencias Sociales, Humanidades y Relaciones Internacionales, realizado en el Centro de Estudios Trasandinos y Latinoamericanos de la Facultad de Ciencias Políticas y Sociales, Universidad Nacional de Cuyo el 9, 10 y 11 de marzo de 2016.

Agradecemos al profesor Víctor Rojas de la Universidad Bernardo O'Higgins por facilitarnos un extracto de la entrevista inédita que realizó al profesor Jeffs en el transcurso del 2015. 


\section{Referencias Citadas}

Acta de Buenos Aires.

2015 Síntesis de la propuesta para una salida al mar de Bolivia. Buenos Aires: Pontificia Universidad Católica Argentina (3 de marzo) http://www.uca.edu.ar/uca/common/grupo56/ files/2015-Prensa-Simposio-Bolivia-Acta-Buenos-Aires. $p d f$ (29 diciembre 2015).

Allard R.

2015 El sistema internacional y América Latina a 50 años del Concilio Vaticano II y de sus propuestas sobre la comunidad entre los pueblos. Revista Estudios Internacionales 47/182.

Boggia, A. \& G. Bravo.

2016 Homenaje a Leonardo Jeffs. Acto de cierre en XIII Seminario Argentino Chileno y VI Seminario Cono Sur de Ciencias Sociales, Humanidades y Relaciones Internacionales. Mendoza: Centro de Estudios Trasandinos y Latinoamericanos de la Facultad de Ciencias Políticas y Sociales, Universidad Nacional de Cuyo.

Bolívar, G. y Ó. Cuéllar.

2012 Hacia la idea de la "Patria Grande". Un ensayo para el análisis de las representaciones políticas. Revista Polis $18 / 2007$.

Cavieres E. y F. Cajías (Coord.).

2008 Chile-Bolivia y Bolivia-Chile: 1820-1930, Ediciones Universitarias de Valparaíso, Valparaíso, Chile.

Centro de Estudios Chilenos.

(12 de diciembre de 2015) http://www.cedech.cl/index. php/78-estable/71-nosotros (26 julio 2016).

Costadoat, S.J., J.

2007 Los "signos de los tiempos" en la Teología de la liberación. Revista Teología y vida 48/4.

Echeverría M.

1993 Clotario Blest. Antihistoria de un luchador 1823-1990, LOM, Santiago, Chile.

Espinoza M.

2005 "Actividades realizadas antes de la creación del Centro de Estudios Latinoamericanos (CEL), Estudios Latinoamericanos, Año 1, $\mathrm{N}^{\circ}$ 1, Segundo semestre 2005, Universidad de Valparaíso.

Fernández D.

1996 Por una iglesia junto al pueblo y sus luchas: El movimiento Iglesia Joven en Santiago de Chile. Anales de la Universidad de Cádiz 11.

Godoy, P.

2015 Homenaje a Leonardo Jeffs. Tercera Línea (3 de marzo de 2016) http://www.terceralinea.cl/index. php? option $=$ com_content $\&$ view $=$ article $\& i d=210:$ leonar do-jeffss \& catid $=88 \&$ Itemid $=1953$ (29 agosto 2016).

González, S. y C. Ovando

2016 Emotivistas bolivianos en la relación diplomática entre Bolivia y Chile en torno a la mediterraneidad. Revista de Estudios Internacionales 183.

Jeffs, L.

1974 Historia económica de Chile: población y precios en el siglo XVIII. Tesis para optar al título de Profesor de Estado en Historia y Geografía, Universidad de Chile, Santiago, Chile. Jeffs, L.

1982 Teoría y práctica del aprismo sobre el imperialismo y la unidad de América Latina, Editorial Nuestra América, Santiago.
Jeffs, L.

1982 Teoría y Práctica del aprismo sobre el imperialismo y la unidad de América Latina. Tesis para optar al grado de Magíster en Relaciones Internacionales, Universidad de Chile, Santiago, Chile.

Jeffs, L.

1995 Aquiles Vergara Vicuña. Perfil biográfico de un hombre íntegro, LOM, Santiago, Chile.

Jeffs, L.

2005 Encuentros y desencuentros: Chile y Bolivia 1928-1935, Peña Andina, Santiago, Chile.

Jeffs, L.

2006 Los sectores medios se han ido alejando de Evo Morales, El Mostrador, 16 de octubre de 2006 (16 de agosto de 2016) http://www.elmostrador.cl/noticias/pais/2006/10/16/ leonardo-jeffs-los-sectores-medios-se-han-ido-alejando-deevo-morales/ (22 diciembre 2015).

Jeffs, L.

2011 Chile y la Guerra del Chaco. Tesis para optar al grado de doctor en Estudios americanos, menciones relaciones internacionales, Universidad de Santiago de Chile, Santiago, Chile.

Jeffs, L.

2012 Encuentros con Bolivia, Peña Andina, Santiago, Chile. Jeffs, L.

2012 La crisis del catolicismo mendocino en los años 1965 y 1966 y sus vinculaciones y repercusiones en la iglesia de Chile. Ponencia presentada en III Jornadas sobre catolicismo y sociedad de masas en la Argentina del siglo XX. Rosario: Universidad de Rosario (12 febrero de 2016) http://historiauv.cl/wp-content/uploads/ LA-CRISIS-DEL-CATOLICISMO-MENDOCINO-EN-LOSA\%C3\%91OS-1965-1966-Y-SUS-VINCULACIONES-YREPERCUSIONES-EN-LA-IGLESIA-DE-CHILE.pdf (22 diciembre 2015).

Jeffs, L.

2012 Las relaciones chileno-bolivianas: aproximación histórica y desafíos. Ponencia presentada en VI Congreso de relaciones internacionales. Instituto de relaciones internacionales, Universidad Nacional de La Plata (16 de agosto de 2016) http://sedici.unlp.edu.ar/bitstream/ handle/10915/40193/Documento_completo.pdf?sequence $=1$ (22 diciembre 2015).

Jeffs, L.

2012 Reflexión sobre la Iglesia Joven (16 agosto) http:// historiauv.cl/wp-content/uploads/REFLEXION-SOBRELA-IGLESIA-JOVEN.pdf (22 diciembre 2015).

Jeffs, L.

2014 La historia de las relaciones internacionales: una experiencia personal (16 de agosto de 2016) http://historiauv.cl/ wp-content/uploads/LA-HISTORIA-DE-LAS-RELACIONESINTERNACIONALES.pdf (22 diciembre 2015).

Jeffs, L.

2014 Mar para Bolivia. Patria Grande, Revista mensual de la izquierda nacional, 6/65.

Lara, $\mathrm{H}$.

2015 Leonardo Jeffs Castro y la misión del profeta. Santiago (16 de agosto de 2016) http://www.g80.cl/noticias/columna_completa.php?varid=21334 (22 diciembre 2015). 
Martí, J.

1975 [1897] Obras Completas, tomo VI, Editorial de Ciencias Sociales, La Habana, Cuba.

Morales, J.J.

2012 De los aspectos sociales del desarrollo económico a la Teoría de la Dependencia: Sobre la gestación de un pensamiento social propio en Latinoamérica. Cinta Moebio 45.

Parra, P.

2002-2003 Leonardo Jeffs: Vocación latinoamericanista. Viajar, arte de magia para la integración. Revista de Estudios Trasandinos 8-9.

Paz Rada, E.

2015 "Leonardo Jeffs: Maestro de la Patria Grande", en $\mathrm{La}$ Época, septiembre de 2015 (16 de agosto de 2015) http:// la-epoca.com.bo/index.php?opt=front \& mod $=$ detalle \& $i d=4879$ (22 diciembre 2015).

Radio Cooperativa.

2015 Académico explicó propuesta de recurrir a Mujica como mediador entre Chile y Bolivia (16 de agosto de 2016) http://www.cooperativa.cl/noticias/pais/ academico-explico-propuesta-de-recurrir-a-mujica-comomediador-entre/2015-05-25/203629.html (22 diciembre 2015).
Rojas, V.

2015 Entrevista inédita a Leonardo Jeffs.

Saladino, A. y A. Santana (Comp.)

2003 Visión de América Latina. Homenaje a Leopoldo Zea, Instituto Panamericano de Geografía e Historia-Fondo de Cultura Económica, Ciudad de México, México.

Salazar, F.

2006 Bolivia y Chile: Desatando nudos. Propuesta de salida al mar para Bolivia basada en el equilibrio de intereses y una nueva racionalidad política, Plural Editores, La Paz, Bolivia.

Soliz Rada, A.

2013 La inagotable persistencia de Leonardo Jeffs: Chile y Bolivia: ¿amistad imposible? Chile/Bolivia. Espacio europeo (16 de agosto de 2016) http://espacioseuropeos. com/2013/10/la-inagotable-persistencia-de-leonardo-jeffschile-y-bolivia-amistad-imposible/ (09 marzo 2016).

Universidad Técnica Federico Santa María.

2015 Expertos se reúnen en las VI Jornadas de Historia de las Relaciones Internacionales en la USM (16 de agosto de 2016) http://humanisticos.usm.cl/2016/03/08/ expertos-se-reunen-en-las-vi-jornadas-de-historia-de-lasrelaciones-internacionales-en-la-usm/ (22 enero 2016).

\section{Notas}

1 Su tesis para optar al título de Profesor de Estado en Historia y Geografía que se titula Historia económica de Chile: población y precios en el siglo XVIII (1974) fue dirigida por el profesor Rolando Mellafe en el Instituto Pedagógico de la Universidad de Chile. Esta investigación fue concluida con varios años de desfase, a causa que una vez egresado partió a trabajar a la región de Valparaíso. En el intertanto, junto con dedicarse a actividades sociales y educacionales, fue candidato a diputado en las elecciones parlamentarias de 1973 para la 2a Agrupación Departamental de Antofagasta, donde obtuvo un discreto resultado.

2 La tesis se desarrolló en el Instituto de Estudios Avanzados de la Universidad de Santiago de Chile, bajo la dirección de la profesora Olga Ulianova.

3 Si bien anteceden obras como las de Jaime Eyzaguirre, Sergio Carrasco y otros, estos trabajos se ajustaban fundamentalmente a la cuestión de límites desde una dimensión histórico-jurídica. Con posterioridad a las investigaciones pioneras de Jeffs, han aparecido interesantes propuestas (Salazar 2006 y Cavieres y Cajías 2008) que se han caracterizado por ser voluntariosos y cargados de buenas intenciones, pero distan de dar propuestas reales en torno al tema de la integración social y cultural de los dos países.

4 Nos referimos a las Jornadas Chileno-Argentina de estudios históricos e integración cultural, organizado por la Asociación homónima de la cual fue cofundador en el año 1995 y Vicepresidente de la entidad.

5 Consciente del peso conceptual y del cambio epistemológico para la Iglesia de la década de los 60, el profesor Jeffs como sujeto histórico, se apropió de la expresión de Juan XXIII, dándole un sentido existencial y trascendente. Para más detalles acerca de la importancia conceptual de la idea, ver el artículo de Costadoat sj, 2007: 407. 